\title{
CARACTERIZAÇÃO DE PROPRIEDADES TECNOLÓGICAS DE PAPÉIS DE FIBRA VIRGEM E RECICLADA APÓS REFINAMENTO
}

\author{
DIFFERENCE OF TECHNOLOGICAL PROPERTIES OF PAPER OF VIRGIN FIBER AND \\ RECYCLED AFTER REFINING \\ Henrique Römer Schulz ${ }^{1}$, Kelvin Techera Barbosa², Andrey Pereira Acosta Cardoso ${ }^{3}$, \\ Gabriel Valim Cardoso 4 \\ 1, 2, 3, 4 Universidade Federal De Pelotas, Pelotas, Rio Grande do Sul, Brasil - \\ henriqueschulz09@hotmail.com, kelvintecherabarbosa@gmail.com,andreysvp@gmail.com \& \\ gabriel.valim.cardoso@gmail.com
}

\begin{abstract}
RESUMO
Um dos processos que modificam as propriedades tecnológicas do papel é o refino, podendo este proporcionar mudanças intra e intermoleculares, o que acarreta aumento de sua resistência mecânica, variando de acordo com o tipo de fibra que se trabalha. Com isso, o objetivo deste estudo é avaliar os efeitos de diferentes graus de refino sobre as propriedades tecnológicas de papéis produzidos com fibras recicladas e virgens de celulose branqueada de Eucalyptus ssp. A produção e caracterização das propriedades tecnológicas foram feitas seguindo técnicas normativas onde verificou-se a influência na gramatura, espessura, densidade volume específico, Grau Schopper Reigler, resistência ao estouro, resistência a tração e elongamento. Ficou evidenciado que com o aumento do grau do refino, houve um aumento nas propriedades físicas e mecânicas, tanto dos papéis produzidos com fibra virgem como reciclada. Com o uso da correlação de Pearson pode-se constatar que determinadas propriedades possuem uma correlação significativa e diretamente proporcional a um nível de $95 \%$ de confiança. Pode-se concluir que a técnica do refinamento faz com que ocorra notoriamente uma melhora nas propriedades físicas e mecânicas de papéis produzidos com fibra virgem e reciclada, apesar do papel reciclado demostrar menores propriedades físicas resultou em melhores resultados em suas propriedades mecânicas com o aumento do grau de refino.
\end{abstract}

PALAVRAS-CHAVE: Eucalyptus, Fibra curta, Propriedades do papel, Refino.

\section{ABSTRACT}

One of the processes that modify the technological properties of the paper is refining, which can provide intra and intermolecular changes which leads to an increase in its mechanical strength, varying according to the type of fiber being worked. The purpose of this study was to evaluate the effects of different grades of refining on the technological properties of papers produced with recycled fibers and virgin bleached cellulose fibers from Eucalyptus ssp. The production and characterization of the technological properties were done following normative techniques where it was verified the influence in weight, thickness, density, specific volume, Schopper Reigler Degree, bursting resistance, tensile strength and elongation. It was evidenced that with the increase of the degree of refining, there was an increase in the physical and mechanical properties, both papers produced with virgin and recycled fiber. Using the Pearson's correlation, certain properties have a significant and directly proportional correlation at a $95 \%$ confidence level. It can be concluded that the refinement technique causes an improvement in the physical and mechanical properties of papers produced with virgin and recycled fiber, and the recycled fiber, although showing lower physical properties, although the recycled paper resulted in better results in its mechanical properties with the increase of the degree of refining.

KEYWORDS: Eucalyptus, Short fiber, Paper properties, Refining. 


\section{INTRODUÇÃO}

No Brasil, o setor de papel e celulose possui grande destaque no setor industrial. Em 2016, o país foi o quarto maior produtor mundial de celulose, como também o nono maior produtor mundial de papel e papelão (DEPEC, 2017). A principal matéria-prima fibrosa para produção de papel é proveniente de plantios do gênero de Eucalyptus, sendo formado por diferentes espécies de rápido crescimento, com grande potencial no setor celulósico (BATISTA, 2010; FORRESTER et al., 2012).

Com o aumento do consumo de papel se faz necessário uma maior produção, ganhando destaque o setor de papéis reciclados. Sob uma perspectiva ambiental tem aumentado a produção destes. $O$ reaproveitamento de materiais já utilizados viabiliza o uso de matéria-prima de menor valor agregado e o desenvolvimento de novas tecnologias que visam otimização desse produto (MANFREDI, 2010; LOMASSO, 2015).

Pelo fato de diminuir o impacto ambiental de produção de lixo, a produção de papéis reciclados tem uma importante participação, retirando toneladas de resíduos e transformando em um importante produto para a indústria nacional (CARDOSO, 2012). Entretanto, esse material muitas vezes necessita de modificação na sua estrutura para se alcançar propriedades físico-mecânicas satisfatórias.

Um dos processos de grande importância que visa o melhoramento de propriedades físicas e mecânicas é o refino. Este é uma etapa importante na produção de papel, pois durante este processo, a aplicação de um trabalho mecânico em um meio aquoso faz com que a morfologia das fibras e sua estrutura física e química sejam modificadas e o papel adquira propriedades (D'ALMEIDA, 1988; GHAREHKHANI, 2015).

Manfredi et al. (2012) e Potulski et al. (2018) constatam que com o refino da polpa celulósica ocorre modificações intra e intermoleculares, o que causa modificações na resistência físico-mecânica de papéis produzidos.

Assim, o objetivo do estudo foi comparar os efeitos de diferentes graus de refino sobre as propriedades tecnológicas, de papéis produzidos com fibras recicladas e virgens de celulose branqueadas de Eucalyptus grandis Ex. Maiden.

\section{MATERIAL E MÉTODOS}

\section{Produção dos papéis}

Para a produção de papel reciclado e de fibras virgens utilizou-se a espécie Eucalyptus grandis. Pesou-se 400 gramas secas de cada tipo de material fibroso, diluídas em dois litros de água destilada separadamente, permanecendo imerso durante 4 horas a fim de hidratar as fibras. As soluções foram transferidas para o desintegrador automático REGMED, modelo DSG-21 de acordo com as normas técnicas ISO 5263-1 e ISO 5263-2. Permanecendo por 10.000 rotações para desagregação completa das fibras. Após o desintegrador foram adicionados 20 litros de água destilada para alcançar $2 \%$ de consistência.

Para formação de folhas sem refino (Passe 0), mediramse $100 \mathrm{ml}$ das soluções fibrosas. Levadas para o equipamento formador, desse passo foi obtido cinco folhas de cada amostra de fibras totalizando dez folhas de acordo com a norma ISO 5269-1. Em seguida, o material foi encaminhado para a prensa pneumática de folhas, modelo SP-21, de acordo com a ISO 5269-1, e posteriormente acondicionado em câmara climatizada segunda a norma ISO 187:1990.

\section{Refino dos papéis}

Foram produzidas 10 folhas no total, sendo 5 de papéis de fibra virgem e 5 de fibras recicladas, submetidas a diferentes graus de refino (Tabela 1 ).

Tabela 1. Refino de fibras virgens e fibras recicladas.

\begin{tabular}{cccccc}
\hline Quantidade de & & $\mathbf{T}$ & CE & \multicolumn{2}{c}{$\mathbf{A}(\mathbf{k W h})$} \\
\cline { 5 - 7 } papéis (PFV/PFR) & $\mathbf{P}$ & (min) & (discos) & FV & FR \\
\hline 2 Folhas & 1 & 1 & $\begin{array}{c}\text { Volta } \\
\text { aberta }\end{array}$ & $2 . .300$ & 2.000 \\
2 Folhas & 2 & 1 & 1 volta & 2400 & 2.100 \\
2 Folhas & 3 & 1 & 2 voltas & 2.500 & 2.500 \\
2 Folhas & 4 & 1 & 3 voltas & 3.200 & 2.600 \\
2 Folhas & 5 & 2 & 3 voltas & 3.400 & 2.700 \\
\hline
\end{tabular}

Em que: $\mathrm{P}=$ passes; $\mathrm{T}=$ tempo; $\mathrm{PFV}=$ papéis de fibras virgens; $\mathrm{CE}=$ condição do equipamento; $\mathrm{PFR}=$ papéis de fibras recicladas; e $\mathrm{A}=$ amperagem.

Para cada grau de refino foram retiradas amostras de $100 \mathrm{ml}$ do refinador, completando até $1000 \mathrm{ml}$ com água destilada, passando assim pelo medidor de Grau de Refino Schopper - Riegler, modelo SR/P, de acordo com a norma ISO 5267-1.

\section{Caracterização de propriedades tecnológicas}

Dos papéis produzidos, foram confeccionadas amostras, nos quais foram determinadas de acordo com cada norma indicada para o desenvolvimento, das 
propriedades mecânicas de estouro (ISO 2758: 2001), espessura (ISO 534: 2005), tração (ISO 1924-2: 2008), elongamento (ISO 1924-3: 2005), gramatura (ISO 536: 1995).

A densidade foi determinada de acordo com a equação (1) e o volume específico conforme a equação (2), exemplificada abaixo.

$$
\begin{aligned}
D & =\frac{(G)}{(E)} \\
V E & =\frac{(E)}{(g)}
\end{aligned}
$$

Em que: $\mathrm{D}=$ densidade do material; $\mathrm{G}=$ gramatura do material; $E$ = espessura do material; e $V E=$ Volume específico.

\section{Análise estatística}

Após os testes tecnológicos, foram efetuadas análises estatísticas visando a homogeneização de dados, com a utilização do diagrama de extremos e quartis, bem como analisadas as médias e coeficiente de variação, posteriormente feita a correlação de Pearson, utilizando os valores médios de cada variável, com $95 \%$ de confiança.

\section{RESULTADOS E DISCUSSÃO}

Os resultados das propriedades físicas do papel produzido com fibra virgem de madeira de Eucalyptus ssp são demostrados na Tabela 2. Evidenciou-se uma heterogeneidade no grupo amostral, com coeficientes de variação de $11,76 \%$ para gramatura, $15,51 \%$ para a densidade, de $16,94 \%$ para o volume específico e a espessura com $5,29 \%$.

Tabela 2. Propriedades físicas do papel produzido com fibra virgem.

\begin{tabular}{cccccc}
\hline $\mathbf{A}$ & $\mathbf{G}\left(\mathbf{g} / \mathbf{m}^{\mathbf{2}}\right)$ & $\mathbf{E}(\mathbf{m m})$ & $\mathbf{D}\left(\mathbf{g} / \mathbf{c m}^{\mathbf{3}}\right)$ & $\mathbf{V e}\left(\mathbf{c m}^{\mathbf{3}} \mathbf{g}\right)$ & $\mathbf{o S R}$ \\
\hline PFV-0 & 41 & 129,79 & 0,313 & 3,198 & 15 \\
PFV-1 & 42 & 128,83 & 0,324 & 3,082 & 15 \\
PFV-2 & 54 & 127,05 & 0,423 & 2,364 & 19 \\
\hline PFV-3 & 50 & 119,98 & 0,416 & 2,402 & 20 \\
PFV-4 & 51 & 115,78 & 0,440 & 2,272 & 21 \\
\hline PFV-5 & 53 & 115,65 & 0,457 & 2,190 & 33 \\
\hline Média & 48 & 122,84 & 0,3955 & 2,585 & 19,66 \\
\hline CV(\%) & 11,76 & 5,29 & 15,51 & 16,94 & 24,42 \\
\hline
\end{tabular}

Em que: $\mathrm{A}=$ amostra; $\mathrm{G}=$ gramatura; $\mathrm{E}=$ espessura; $\mathrm{Ve}=$ volume específico; PFV-0= Passe 0; PFV-1= Passe $1 ; \mathrm{PFV}-2=$ Passe 2; PFV3= Passe 3; PFV-4= Passe 4; PFV-5= Passe 5; CV = coeficiente de variação; e oSR = Grau Schopper Riegler.
Pode-se observar que com o aumento do grau do refino, houve um aumento na densidade e na gramatura. Zanuncio et al. (2016) explica que o esforço do refino causa o achatamento do material fibroso, reduzindo o volume antes ocupado pelo lume da fibra e com isso aumentando a densidade do papel produzido. A amostra (PFV-0) apresentou o menor valor de densidade, a menor gramatura e o maior valor de volume específico. A amostra (PFV-5), apresentou o maior valor de densidade, obtendo um aumento de 46,07\%, o segundo maior valor de gramatura, com aumento de $29,27 \%$ e o menor volume específico, com 31,52 \% de redução, comparado a amostra de fibra virgem sem passe (PFV-0), isto se justifica pelo fato de que quanto maior o passe há maior entrelaçamento das fibras e isso acarreta em uma maior densidade, menor volume e maior gramatura.

Constata-se que o refino interfere nas propriedades físicas do papel, aumentando a densidade e a gramatura, e diminuindo o volume específico. Gatti (2007) evidência em sua pesquisa que o refino é um tratamento mecânico no qual as fibras da polpa de celulose são rompidas em fibrilas, aumentando, assim, a área superficial, o que por consequência eleva as propriedades mecânicas do papel.

As propriedades físicas do papel produzido de fibra reciclada são demostradas na Tabela 3, sendo observada a diferença nos valores no grupo amostral obtendo coeficientes de variação de 7,66\% para gramatura, 7,85\% para a densidade, de $8,28 \%$ para o volume específico e a espessura com $0,51 \%$.

Tabela 3. Propriedades físicas do papel produzido com fibra reciclada.

\begin{tabular}{cccccc}
\hline $\mathbf{A}$ & $\mathbf{G}\left(\mathbf{g} / \mathbf{m}^{\mathbf{2}}\right)$ & $\mathbf{E}(\mathbf{m m})$ & $\mathbf{D}\left(\mathbf{g} / \mathbf{c m}^{\mathbf{3}}\right)$ & $\mathbf{V e}\left(\mathbf{c m}^{\mathbf{3}} \mathbf{g}\right)$ & $\mathbf{o S R}$ \\
\hline PFR-0 & 39 & 86,48 & 0,411 & 2,217 & 28 \\
PFR-1 & 33 & 87,68 & 0,372 & 2,687 & 31 \\
PFR-2 & 39 & 87,23 & 0,444 & 2,253 & 34 \\
PFR-3 & 38 & 86,98 & 0,350 & 2,299 & 37 \\
PFR-4 & 38 & 86,83 & 0,436 & 2,292 & 41 \\
PFR-5 & 33 & 86,58 & 0,386 & 2,591 & 50 \\
\hline Média & 37,00 & 86,960 & 0,421 & 2,390 & 36,83 \\
\hline CV(\%) & 7,66 & 0,51 & 7,85 & 8,28 & 13,03 \\
\hline
\end{tabular}

Em que: $\mathrm{A}=$ amostra; $\mathrm{G}=$ gramatura; $\mathrm{E}=$ espessura; $\mathrm{Ve}=$ volume específico; PFV-0= Passe 0; PFV-1= Passe $1 ; \mathrm{PFV}-2=$ Passe $2 ; \mathrm{PFV}$ 3= Passe 3; PFV-4= Passe 4; PFV-5= Passe 5; CV = coeficiente de variação; e @SR = Grau Schopper Riegler.

Em relação as propriedades físicas do papel com fibra reciclada, a gramatura e a densidade para a as amostras (PFR-2) e (PFR-4), apresentaram os maiores valores de densidade e gramatura, com um aumento de $8,02 \%$ e 
$6,08 \%$ quando comparado a amostra sem refino com passe 0 (PFR-0). Para o ${ }^{\circ} \mathrm{SR}$, conforme o aumento no grau de refinação, ocorreu uma tendência de aumento.

As amostras não demonstraram uma tendência de aumento para as propriedades físicas conforme o aumento do refino, tal fato, é em função de ser utilizadas fibras que já sofreram um processamento (fibras recicladas), como também em função da degradação das fibras com um refino severo. Segura et al. (2010) e Olejnik (2013) citam que as propriedades tecnológicas dos papéis crescem com o aumento do grau de refino, até certo momento, e passa a decrescer gradativamente quando o grau excede, pois ocorre a degradação severa nas fibras.

A Tabela 4 apresenta os resultados de propriedades mecânicas do papel produzido de fibra virgem de madeira de Eucalyptus ssp., sendo analisada uma diferença nos valores no grupo amostral obtendo-se de coeficientes de variação de 7,61\% para resistência ao estouro, 36,85\% para a resistência a tração e de $16,28 \%$ para o elongamento.

Tabela 4. Propriedades mecânicas do papel produzido com fibra virgem.

\begin{tabular}{ccccc}
\hline A & RE (KPa.m $\mathbf{2} / \mathbf{g})$ & RT (KN.m/kg) & E (\%) & oSR \\
\hline PFV-0 & 1,318 & 14,34 & 1,53 & 15 \\
\hline PFV-1 & 1,328 & 15,05 & 1,57 & 15 \\
\hline PFV-2 & 1,358 & 15,96 & 1,59 & 19 \\
\hline PFV-3 & 1,392 & 17,50 & 1,63 & 20 \\
\hline PFV-4 & 1,540 & 23,94 & 1,65 & 21 \\
\hline PFV-5 & 1,562 & 33,33 & 2,26 & 33 \\
\hline Média & 1,416 & 20,02 & 1,70 & 19,66 \\
\hline CV (\%) & 7,61 & 36,85 & 16,28 & 24,42 \\
\hline
\end{tabular}

Em que: $\mathrm{A}=$ amostra; $\mathrm{G}=$ gramatura; $\mathrm{E}=$ espessura; $\mathrm{Ve}=$ volume específico; PFV-0= Passe 0; PFV-1= Passe 1; PFV-2= Passe 2; PFV3= Passe 3; PFV-4= Passe 4; PFV-5= Passe 5; CV = coeficiente de variação; e oSR = Grau Schopper Riegler.

Observa-se que as propriedades mecânicas dos papéis de fibra virgem, a amostra (PFV-0) apresentou os menores valores e a amostra (PFV-5) apresentou os maiores valores, para a resistência ao estouro apresentou um aumento de, $18,51 \%$, para resistência a tração $132,43 \%$, o elongamento $47,71 \%$ e o ${ }^{\circ}$ SR $120 \%$.

Silva (2000) e Manfredi et al. (2012) constatam que a refinação está diretamente ligada com as propriedades físico-mecânicas do papel, sendo diversos os efeitos para as fibras, com a fibrilação externa, ocorrem a formação de filamentos superficiais que aumentam o entrelaçamento entre as fibras do papel e a fibrilação interna, promovendo aumento da flexibilidade das fibras, consequentemente aumentando a resistência.

Sendo assim, o refino causa uma melhor distribuição e homogeneização da polpa celulósica, proporcionando um aumento na flexibilidade das fibras e evitando a formação de aglomerados. Interferindo nas propriedades mecânicas do papel, aumentando os valores de suas propriedades, devido ao melhor entrelaçamento das fibras.

Carvalho (2015) constata que no refino prolongado pode ocorrer, a formação de finos causada pelo destacamento total de partículas das paredes primária e secundária das fibras, como também o encurtamento de fibras, causado pelas lâminas do refinador. Sendo tais parâmetros indesejáveis, causando perda de resistência físico-mecânica. Segundo (GIGAC \& FIŠEROVÁ, 2008), o refino deve se ser feito até um ponto de equilíbrio, visando melhorias nas propriedades físico-mecânicas.

A Tabela 5 apresenta os resultados de propriedades mecânicas do papel produzido com fibra reciclada, sendo evidenciada uma diferença nos valores no grupo amostral obtendo se coeficientes de variação de 15,93\% para resistência ao estouro, 16,07\% para a resistência a tração, de $18,23 \%$ para o elongamento e para o Grau de Refino Schopper - Riegler (ㅇR) de 13,03\%.

Tabela 5. Propriedades mecânicas do papel produzido com fibra reciclada.

\begin{tabular}{ccccc}
\hline A & RE (KPa. $\left.\mathbf{m}^{\mathbf{2}} \mathbf{g}\right)$ & $\mathbf{R T}$ (KN. $\mathbf{~ m / k g )}$ & $\mathbf{E}$ (\%) & oSR \\
\hline PFR-0 & 1,564 & 23,47 & 2,42 & 28 \\
PFR-1 & 1,701 & 32,89 & 2,65 & 31 \\
PFR-2 & 2,157 & 34,09 & 3,29 & 34 \\
PFR-3 & 2,154 & 35,42 & 3,46 & 37 \\
PFR-4 & 2,178 & 36,69 & 3,77 & 41 \\
\hline PFR-5 & 2,409 & 39,05 & 3,87 & 50 \\
\hline Média & 2,027 & 33,60 & 3,24 & 36,83 \\
\hline CV (\%) & 15,93 & 16,07 & 18,23 & 13,03 \\
\hline
\end{tabular}

Em que: $\mathrm{A}=$ amostra; $\mathrm{G}=$ gramatura; $\mathrm{E}=$ espessura; $\mathrm{Ve}=$ volume específico; PFV-0= Passe 0; PFV-1= Passe 1; PFV-2= Passe 2; PFV3= Passe 3; PFV-4= Passe 4; PFV-5= Passe 5; CV = coeficiente de variação; e oSR = Grau Schopper Riegler.

As propriedades mecânicas de papéis de fibra reciclada, apresentaram uma tendência de aumento conforme o aumento do passe do refino. Destacando a amostra (PFR$5)$, que apresentou um acréscimo de $54,02 \%$ a resistência ao estouro, resistência a tração $66,38 \%$, elongamento $59,92 \%$ e oSR $78,57 \%$, quando comparada a amostra sem refino (PFR-0).

As propriedades mecânicas aumentaram seu valor conforme aumentou o refino. A resistência ao rasgo é uma 
propriedade influenciada por diversos fatores, dentre os quais se destacam espessura, orientação das fibras, comprimento e rigidez, destacando o grau de refinação (SIMISTRARO, 2009).

A resistência ao estouro e a resistência à tração são influenciadas pelo comprimento das fibras e, principalmente, pelos efeitos mecânicos advindos do processo de refino, quando há o colapso da parede celular, aumentando assim sua área de contato (BIERMANN, 1996).

Comparando o refino sobre as propriedades mecânicas entre os tipos de fibras analisadas percebe-se que este processo possui maior influência sobre papéis com fibra reciclada, as quais demostraram valores mais elevados em relação a resistência mecânica, isto se justifica pelo passo deste tipo de fibra já ter passado por um processo mecânico de refinamento, fazendo assim que as fibras fiquem com um entrelaçamento mais elevado que a fibra virgem.

A Tabela 6 apresenta a correlação de Pearson para as propriedades físicas, densidade $(\rho)$, espessura, gramatura, oSR e volume específico de fibra virgem e fibra reciclada Eucalyptus ssp.

Tabela 6. Correlação de Pearson para propriedades físicas.

\begin{tabular}{|c|c|c|c|c|c|}
\hline & $\rho$ & $\mathbf{E}$ & $\mathbf{G}$ & OSR & Ve \\
\hline \multicolumn{6}{|c|}{ Papel produzido com fibra virgem } \\
\hline$\rho$ & 1 & $0,8513^{*}$ & $0,9595^{*}$ & 0,7778 & $-0,9979 *$ \\
\hline$E$ & & 1 & $-0,6693$ & $-0,7863$ & $0,8339 *$ \\
\hline G & & & 1 & 0,675 & $-0,9664 *$ \\
\hline oSR & & & & 1 & $-0,7427$ \\
\hline Ve & & & & & 1 \\
\hline \multicolumn{6}{|c|}{ Papel produzido com fibra reciclada } \\
\hline$\rho$ & 1 & $-0,3521$ & 0,5829 & $-0,2705$ & $-0,5924$ \\
\hline$E$ & & 1 & $-0,2954$ & $-0,3584$ & 0,4442 \\
\hline G & & 1 & 1 & $-0,4264$ & $-0,9861 *$ \\
\hline oSR & & & & 1 & 0,3077 \\
\hline $\mathrm{Ve}$ & & & & & 1 \\
\hline
\end{tabular}

Em que: $\mathrm{E}=$ espessura; $\mathrm{G}=$ gramatura; $\mathrm{Ve}=$ volume específico; * = significativo com 95\% de confiança; e $\rho=$ densidade.

Os resultados obtidos para as propriedades físicas do papel feito por fibras virgem apresentaram maiores correlações significativas em relação ao papel de fibra reciclada, pois a fibra reciclada é um material de reprocessamento. MANFREDI et al., 2012, relata que as fibras recicladas já processadas mecanicamente, apresentam menores valores das propriedades físicas do papel.

Constatou-se correlações significativas para a densidade com a gramatura sendo diretamente proporcional, e inversamente proporcional com a espessura e o volume específico, para os papéis de fibra virgem. Quando analisado os papéis de fibra reciclada, somente a gramatura teve correlação significativa, sendo inversamente proporcional com ao volume específico.

A Tabela 7 apresenta a correlação de Pearson para as propriedades mecânicas, elongamento, resistência a tração, resistência ao estouro e oSR de fibra virgem e fibra reciclada Eucalyptus ssp.

Tabela 7. Correlação de Pearson para propriedades mecânicas.

\begin{tabular}{|c|c|c|c|c|}
\hline & $\mathbf{E}$ & RT & RE & oSR \\
\hline \multicolumn{5}{|c|}{ Papel produzido com fibra virgem } \\
\hline$E$ & 1 & $0,9347^{*}$ & 0,7538 & $0,9679 *$ \\
\hline RT & & 1 & $0,936^{*}$ & $0,9582^{*}$ \\
\hline $\mathrm{RE}$ & & & 1 & $0,8435^{*}$ \\
\hline oSR & & & & 1 \\
\hline \multicolumn{5}{|c|}{ Papel produzido com fibra reciclada } \\
\hline$E$ & 1 & $0,8902 *$ & $0,9699 *$ & $0,9042^{*}$ \\
\hline RT & & 1 & $0,8894^{*}$ & $0,8319 *$ \\
\hline $\mathrm{RE}$ & & & 1 & $0,887^{*}$ \\
\hline oSR & & & & 1 \\
\hline
\end{tabular}

Em que: $\mathrm{E}=$ elongamento; $\mathrm{RT}=$ resistência a tração; $\mathrm{RE}=$ resistência ao elongamento; $\mathrm{e}^{*}=$ significativo com $95 \%$ de confiança.

As propriedades mecânicas para ambos os tipos de papéis apresentaram correlações significativas, sendo essas diretamente proporcional, excetuando-se a resistência ao estouro com o elongamento, não sendo significativa. Carvalho (2015), em um estudo relacionado as propriedades físico-mecânicas em papeis, encontrou uma correlação entre as propriedades mecânicas e o grau de refino, onde pode-se destacar índices de tração, elongamento e rasgo, apresentou correlação diretamente proporcional com o nível de refino.

\section{CONCLUSÕES}

Ao se analisar o processo de refino na produção de papéis, tanto de fibra virgem como fibra reciclada, constata-se uma modificação nas propriedades tecnológicas com o aumento do mesmo, geralmente benéficas, destacando as propriedades de resistência ao estouro, resistência a tração, elongamento, ${ }^{\circ} \mathrm{SR}$ e densidade mostrando assim notoriamente um aumento conforme.

Observou-se que determinadas propriedades têm 
correlações significativas, sendo diretamente ou indiretamente proporcional, quando comparada às outras, tanto para mecânicas, como para físicas.

Os papéis produzidos com fibras recicladas e virgens, apresentaram parâmetros consistentes relacionados as propriedades físicos-mecânicas.

\section{REFERÊNCIAS}

ABNT - Associação Brasileira de Normas Técnicas. NBR 14003: Especifica um método para determinar a consistência de pastas celulósicas. 2003.

BATISTA, D.C. et al. Densidade básica e retratibilidade da madeira de clones de três espécies de Eucalyptus. Ciência Florestal, v.20, n.4, p.665-674, 2010.

BIERMANN, C. Handbook of pulping and papermaking. 2.ed. Academic Press, 1996.

CARDOSO, M.T. et al. Propriedades físicas e mecânicas de papéis reciclados utilizados para fabricação de tubetes. Ciência Florestal, v.22, n.2, p.407-415, 2012.

CARVALHO, D. et al. Estudo da relação entre condições de polpação e propriedades físico-mecânicas do papel. Revista Árvore, v.39, n.3, p.575-584, 2015.

D'ALMEIDA, M.L.O. Celulose e papel: tecnologia de fabricação da pasta celulósica. 2.ed. São Paulo: IPT, 1988.

DEPEC - DEPARTAMENTO DE PESQUISA E ESTUDOS ECONÔMICOS. Papel e celulose, 2017.

FORRESTER, D.I.; SMITH, R.G.B. Faster growth of Eucalyptus grandis and Eucalyptus pilularis in mixed-species stands than monocultures. Forest Ecology and Management, v.286, p.81-86, 2012.

GATTI, T.H.A história do papel artesanal no Brasil. ABTCPAssociação Brasileira Técnica de Celulose e Papel. São Paulo, 2007.

GHAREHKHANI, S. et al. Basic effects of pulp refining on fiber properties-a review. Carbohydrate polymers, v.115, p.785-803, 2015.

GIGAC, J.; FIŠEROVÁ, M. Influence of pulp refining on tissue paper properties. Tappi Journal, v.7, n.8, p.27-32, 2008.

LOMASSO, A L. et al. Benefícios e desafios na implementação da reciclagem: um estudo de caso no Centro Mineiro de Referência em Resíduos (CMRR). Revista Pensar Gestão e Administração, v.3, n.2, p.1-20, 2015.

ISO - International Organization for Standardization. ISO-187: Paper, board and pulps - Determination of drainability. 1990.

ISO - International Organization for Standardization. ISO-1924-2: Paper and board - Determination of tensile. 2008.
ISO - International Organization for Standardization. ISO-1924-3: Techstreet. 2005.

ISO - International Organization for Standardization. ISO-2758: Paper - Determination of bursting strength. 2001.

ISO - International Organization for Standardization. ISO-5263-1: Pulps. 2004.

ISO - International Organization for Standardization. ISO-5263-2: Pulps. 2004.

ISO - International Organization for Standardization. ISO-5267-1: Pulps. 1999.

ISO - International Organization for Standardization. ISO-5269-1: Pulps. 2005.

ISO - International Organization for Standardization. ISO-534: Paper and board - Determination of thickness, density. 2005.

ISO - International Organization for Standardization. ISO-536: Paper and board - Determination of grammage. 1995.

MANFREDI, M. Desenvolvimento de propriedades de papéis reciclados por tratamento ultrassônicos e adição de xilanas. 2010. 71 p. (Dissertação de mestrado).

MANFREDI, M. et al. Melhoramento das propriedades de papéis reciclados através da ultrassonificação das fibras e adição de xilanas. Revista Árvore, v.36, p.777-785, 2012.

OLEJNIK, K. Impact of pulp consistency on refining process conducted under constant intensity determined by SEL and SEC factors. BioResources, v.8, n.3, p.3212-3230, 2013.

POTULSKI, D.C. et al. Influência da adição de celulose nanofibrilada (cnf) nas propriedades ópticas e físicas do papel. BIOFIX Scientific Journal, v.1, p.122-129, 2018.

SAMISTRARO, G. et al. Previsão das propriedades físicas do papel Kraft por espectroscopia no infravermelho próximo (NIR) e regressão por mínimos quadrados parciais (PLS). Química Nova, v.32, p.1422-1425, 2009.

SEGURATES, Z.M.; SILVA, F.G. Potencial da Madeira de acácia para a produção de polpa celulósica Kraft. 210 Encontro Nacional da TECNICELPA / VI CIADICYP, p.8, 2010.

SILVA, R.P.; OLIVEIRA, R.C. Efeitos da ação do refino e da reciclagem nas propriedades de papéis de pinus e eucalipto. Revista Árvore, v.24, n.2, p.349-360, 2000.

ZANUNCIO, A.J.V. et al. Avaliação das propriedades físicas, mecânicas e ópticas da polpa celulósica durante refino. Revista Ciência da Madeira, v.7, n.2, p.81-87, 2016. 